REVISTA DE GEOCIÊNCIAS DO NORDESTE

Northeast Geosciences Journal

v. $7, \mathrm{n}^{\circ} 2(2021)$

ISSN: $2447-3359$

https://doi.org/10.21680/2447-3359.2021v7n2ID24543

\title{
VARIÁVEIS METEOROLÓGICAS E O NÚMERO DE INTERNAÇÕES POR INFARTO AGUDO DO MIOCÁRDIO EM BELÉM/PA
}

\begin{abstract}
Ana Vitória Padilha Mendes'; ${ }^{1}$ Arthur Wendell Duarte Silva ${ }^{2}$; Ana Carla dos Santos Gomes ${ }^{3}$ Lucas Vaz Peres ${ }^{4}$ Antonio Marcos Delfino de Andrade $^{5}$
\end{abstract}

${ }^{1}$ Graduando, IEG/Ciências atmosféricas, Universidade Federal do Oeste do Pará (UFOPA), Santarém/PA, Brasil.

ORCID: https://orcid.org/0000-0002-0569-4297

Email: nha_2309@hotmail.com

${ }^{2}$ Graduando, IEG/Ciências atmosféricas, Universidade Federal do Oeste do Pará (UFOPA), Santarém/PA, Brasil.

ORCID: https://orcid.org/0000-0002-1746-3832

Email: arthurweendell@gmail.com

${ }^{3}$ Professora Doutora, IEG/Ciências atmosféricas, Universidade Federal do Oeste do Pará (UFOPA), Santarém/PA, Brasil. ORCID: https://orcid.org/0000-0001-7499-8342

Email: anacarlasg02@gmail.com

${ }^{4}$ Professor Doutor, IEG/Ciências atmosféricas, Universidade Federal do Oeste do Pará (UFOPA), Santarém/PA, Brasil.

ORCID: https://orcid.org/0000-0002-5612-5991

Email: lucasvazperes@gmail.com

${ }^{5}$ Professor Doutor, IEG/Ciências atmosféricas, Universidade Federal do Oeste do Pará (UFOPA), Santarém/PA, Brasil.

ORCID: https://orcid.org/0000-0002-2075-7890

Email: marcoslaba33@gmail.com

\section{Resumo}

O objetivo deste estudo foi verificar se as variáveis meteorológicas influenciam de forma significativa nas causas de morbidade por Infarto agudo do miocárdio em Belém do Pará. Para isso foram utilizados dados de temperatura máxima e mínima do ar, umidade relativa do ar, precipitação pluvial e velocidade do vento, disponibilizados pelo Banco de Dados Meteorológicos para Estudo e Pesquisa do Instituto Nacional de Meteorologia e dados de internações por IAM obtidos através do
Departamento de Informática do Sistema Único de Saúde, ambos no período de 2008 a 2018, totalizando 10 anos de dados, foram registradas 4242 internações durante esse período . Utilizou-se a estatística descritiva e a modelagem via Equações de Estimação Generalizadas para verificar associação, por meio do software livre R 3.4.1. Por fim, os resultados assinalam associação significativa entre a temperatura máxima do ar, velocidade do vento e o número de internações. A sinergia entre o aumento da temperatura máxima e diminuição na velocidade do vento favoreceram para o aumento do número de internações em Belém, acredita-se que os resultados observados possam ser um bom contribuinte para políticas públicas de saúde.

Palavras-chave: Modelagem estatística; Morbidade; Clima.

\section{METEOROLOGICAL VARIABLES AND THE NUMBER OF HOSPITALIZATIONS FOR ACUTE MYOCARDIAL INFARCTION IN BELÉM/PA}

\begin{abstract}
The objective of this study was to verify whether the meteorological variables significantly influence the causes of morbidity due to acute myocardial infarction in Belém do Pará. For this purpose, maximum and minimum air temperature, relative humidity, rainfall and velocity data were used. of the wind, made available by the Meteorological Database for Study and Research of the National Institute of Meteorology and hospitalization data for AMI obtained through the Department of Informatics of the Unified Health System, both in the period from 2008 to 2018 , totaling 10 years of data, 4242 admissions were recorded during this period. Descriptive statistics and modeling using Generalized Estimation Equations were used to verify association, using the free software R 3.4.1. Finally, the results indicate a significant association between maximum air temperature, wind speed and the number of hospitalizations. The synergy between the increase in maximum temperature and decrease in wind speed favored the increase in the number of hospitalizations in Belém, it is believed that the results observed may be a good contributor to public health policies.
\end{abstract}

Keywords: Statistical modeling; Morbidity; Climate. 


\section{VARIABLES METEOROLÓGICAS Y EL NÚMERO DE HOSPITALIZACIONES POR INFARTO AGUDO DE MIOCARDIO EN BELÉM/PA}

\section{Resumen}

El objetivo de este estudio fue verificar si las variables meteorológicas influyen significativamente en las causas de morbilidad por infarto agudo de miocardio en Belém do Pará, para lo cual se utilizaron datos de temperatura máxima y mínima del aire, humedad relativa, precipitación y velocidad. el viento, puesto a disposición por la Base de Datos Meteorológicos de Estudio e Investigación del Instituto Nacional de Meteorología y datos de hospitalización por IAM obtenidos a través del Departamento de Informática del Sistema Único de Salud, ambos en el período de 2008 a 2018, totalizando 10 años de datos, Se registraron 4242 admisiones durante este período. Para verificar la asociación se utilizó estadística descriptiva y modelado mediante Ecuaciones de Estimación Generalizadas, utilizando el software libre R 3.4.1. Finalmente, los resultados indican una asociación significativa entre la temperatura máxima del aire, la velocidad del viento y el número de hospitalizaciones. La sinergia entre el aumento de la temperatura máxima y la disminución de la velocidad del viento favoreció el aumento del número de hospitalizaciones en Belém, se cree que los resultados observados pueden ser un buen contribuyente a las políticas de salud pública.

Palabras-clave: Modelado estadístico; Morbilidad; Clima.

\section{INTRODUÇÃO}

As doenças cardiovasculares podem afetar o coração e os vasos sanguíneos, destacando-se a doença arterial coronariana, que envolve dor no peito e infarto agudo do miocárdio, sendo esta a maior causa de morbimortalidade no mundo. No Brasil, de acordo com o Ministério da Saúde, cerca de 300 mil indivíduos por ano sofrem Infarto Agudo do Miocárdio (IAM), ocorrendo óbito em 30\% desses casos (BRASIL, 2019). No Brasil, o IAM resultam em altas taxas de incidência de mortalidade, chegam em torno de (183,3/ 100.000 habitantes) estando entre as mais elevadas do mundo e é semelhante ao de países como a China e áreas como o Leste da Europa (SANTOS et al, 2018).

Segundo o Ministério da Saúde, o infarto do miocárdio, ou ataque cardíaco, é a morte das células de uma região do músculo do coração por conta da formação de um coágulo que interrompe o fluxo sanguíneo de forma súbita e intensa (MS, 2018). A principal causa do infarto é a aterosclerose, doença em que placas de gordura se acumulam no interior das artérias coronárias, chegando a obstruí-las. Nesses casos, o infarto ocorre quando há o rompimento de uma dessas placas, levando à formação do coágulo e interrupção do fluxo sanguíneo, segundo Bacelar, 2012.

Segundo Medeiros et al., 2018, a alimentação saudável desde a infância é essencial para a saúde e diminui os riscos de Doenças Cardiovaculares no futuro. Os hábitos alimentares da maioria dos jovens mudaram significativamente nas últimas décadas devido ao grande consumo de alimentos com alto teor de gordura, colesterol e carboidrato. Consequentemente, essas pessoas tendem a ter maior risco em adquirir DCV na fase adulta.

Não é novidade que as alterações climáticas produzem efeitos diretos e indiretos na saúde, no entanto, nunca se deu tanta atenção às pesquisas que envolvem os temas relacionados com as variáveis ambientais e a saúde humana. Prova disto são os vários estudos realizados em todo mundo pelos mais diversos profissionais pesquisadores, tanto das ciências exatas como da biologia, formando uma união dentre essas áreas, aumentando o nível de pesquisas com uma interdisciplinaridade, que visa uma melhor entendimento das interelações entre os seres vivos e o meio ambiente. De acordo com Pereira et al., 2012, as mudanças que ocorrem com as variáveis meteorológicas sobre algumas regiões são visadas como um fator bastante preocupante por estudiosos em climatologia e sabe-se que essas mudanças não influenciam somente o microclima dessas regiões, podem ocasionar variações fisiológicas consideráveis no que diz respeito às doenças relacionadas com as variáveis climatológicas.

O estudo das relações entre a Biosfera e a atmosfera, nos dias atuais tornou-se uma ciência cujo fim é prevenir as pessoas em risco, devido à grande influência dos elementos meteorológicos sobre o organismo humano (PEREIRA, 2012). Pois, os efeitos das mudanças do clima também podem incidir de maneira direta ou indireta, gerando consequências físicas, traumáticas, psicológicas, infecciosas e nutricionais (OLIVEIRA, 2019). Segundo (DILAVERIS, et al, 2006) a contribuição das condições climáticas para este fim tem sido reconhecida diante vários estudos que indicam uma variação sazonal das taxas de morbimortalidade da doença cardiovascular.

As mudanças climáticas atingem alterações oriundas de mudanças na temperatura do ar, precipitações, entre outros fenômenos, em relação a médias históricas, de maneira que, acabam interferindo nas características climáticas do planeta (ARAUJO, 2018). Para Lee et al., 2010, nos últimos anos, muita atenção tem sido dada para a variação sazonal do IAM. Distintos avisos indicam que Infarto Agudo do Miocárdio incide mais comumente nas estações frias, especialmente nas zonas temperadas e frias. Com isso, alguns relatórios das áreas subtropicais revelaram que as admissões hospitalares por IAM se elevam durante as estações quentes de verão em comparação as estações de inverno (IKEFUTI, 2017). Dessa forma, o Infarto Agudo do Miocárdio é semelhante ao estresse térmico, isto é, onde as temperaturas chegam em pontos extremamente frios ou quentes. Segundo Goerre et al,. 2007, as primeiras investigações das interações entre clima e da incidência de Infarto Agudo do Miocárdio são desde 1938

Para Morabito et al,. 2005, a combinação de temperatura do ar, umidade relativa e velocidade do vento, fornece valores de temperatura aparentes que permitem uma avaliação do aumento da percepção de desconforto humano causado pela eficiência comprometida da melhor transpiração. No entanto, o efeito de condições de tempo quente pode não ser apreciada considerando apenas as temperaturas médias diárias do ar (MORABITO et al. 2005). Estudos realizados em regiões tropicais relataram que também que o clima quente pode aumentar a incidência de infarto agudo (MORABITO et al. 2005).

No trabalho realizado por Teng e Heyer., 1955, constatou-se que há associação significativa entre a temperatura máxima e o número de casos por IAM na cidade de Dallas, situada nos trópicos, localizada no norte do estado do Texas/EUA. Revelouse que, quando a ocorrência sazonal foi tabulada, o maior número de casos ocorreu durante os meses de verão e o menor número de casos durante o inverno. As ocorrências estão atreladas pela 
entrada de ar tropical, transportadas pelos ventos do sul ou sudeste, causando um aumento na temperatura do ar e havendo uma redução simultânea da pressão barométrica.

Ainda de acordo com Teng e Heyer., 1955, houve casos onde pacientes tiveram início dos sintomas de IAM dentro das suas residências, em um ambiente quente, durante o sono ou em repouso. Segundo Heyer e Teng., 1953, o clima caracterizado por muito calor (clima de verão), o IAM ocorre com mais frequência durante a estação mais quente do ano. Além de influenciar o microclima de regiões, podem ocasionar variações fisiológicas consideráveis no que diz respeito às doenças relacionadas com as variáveis meteorológicas, dessa forma agravando algumas doenças pré-existentes e favorecendo o aparecimento de outras, tornando-se assim um problema de saúde pública (PEREIRA et al., 2007). Diante do exposto, o objetivo deste trabalho é verificar se as variáveis meteorológicas influenciam de forma significativa nas causas de morbidade por Infarto Agudo do Miocárdio em Belém/PA.

\section{METODOLOGIA}

O local de estudo foi o município de Belém, capital do Estado do Pará $\left(01^{\circ} 27^{\prime} 20^{\prime \prime} \mathrm{S}\right.$; 40³0'15" O), que possui uma área territorial de $1.059,458 \mathrm{~km}^{2}$ (Figura 1), situada na zona equatorial, às margens da Baía do Guajará e Rio Guamá, afastada 120 km do Oceano Atlântico (IBGE, 2018). De acordo com a classificação de Koppen-Geiger, Belém tem um clima tropical do tipo Am., com precipitação média anual de $2537 \mathrm{~mm}$. Sendo uma região onde as chuvas são constantes, a cidade apresenta duas estações: estação chuvosa (dezembro a maio) e estação menos chuvosa (junho a novembro), conforme descrito por Nechet (1984), Figueroa e Nobre (1990), Bastos et al., (2002) e Oliveira et al., (2003).

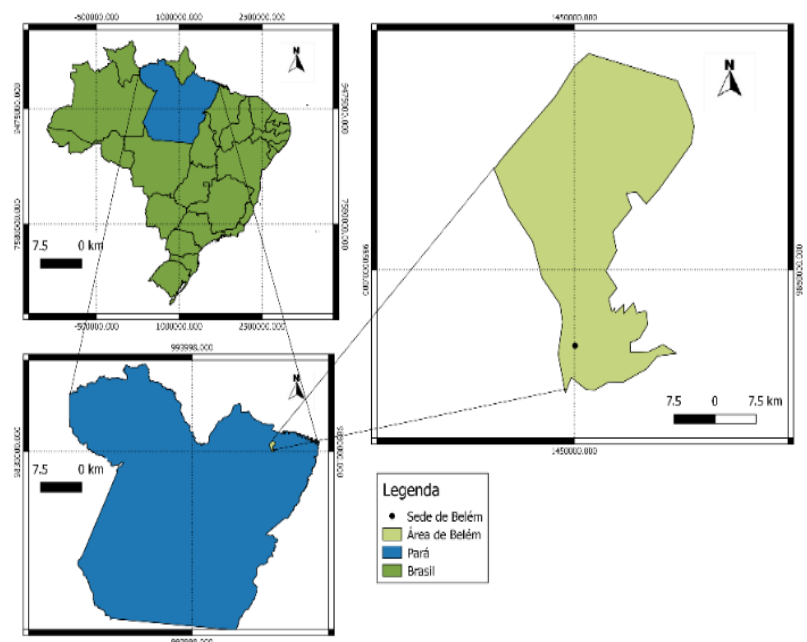

Figura 1 - Localização geográfica da área de estudo. Fonte: IBGE (2019), adaptado pelos autores.

Para a realização deste estudo, foram utilizados dados mensais das variáveis meteorológicas precipitação pluvial (mm), temperatura máxima e mínima do ar $\left({ }^{\circ} \mathrm{C}\right)$, umidade relativa do ar (\%) e velocidade do vento $(\mathrm{m} / \mathrm{s})$ proveniente da estação meteorológica $\left(-1.43^{\circ} \mathrm{S} ;-48.43^{\circ} \mathrm{O} ; 10.00 \mathrm{~m}\right.$ alt.), disponíveis pelo Instituto Nacional de Meteorologia (INMET).

Foram registrados 4182 casos de morbidade por IAM, no período de 2008 a 2018, pelo Departamento de Informática do Sistema Único de Saúde (DATASUS). Desde que o SUS foi implementado, o DATASUS tem por principal objetivo estruturar sistemas de informação em saúde, agregando dados em saúde, bem como facilitando na gestão dos diversos níveis de atenção em saúde (DATASUS, 2019).

Com os dados de séries mensais, obtiveram-se os totais anuais das variáveis. A climatologia é representada por gráficos do tipo Boxplot. O boxplot permite resumir os dados podendo assim, mostrar a mediana que é representada por Q2 ou segundo quartil, localizada dentro do box e valores pontuais representados por Q3 (terceiro quartil) que totaliza $75 \%$ dos valores máximos e os mínimos Q1 (primeiro quartil) representando $25 \%$ desses valores. A haste (bigode ou whisker), representa os valores localizados entre o box e os valores limites, definidos pelas normas de Tukey (Wilks, 2006), seguindo as seguintes expressões:

$$
\begin{aligned}
& x 1=Q 1-1,5 * 1 Q R \\
& X n=Q 3+1,5 * 1 Q R
\end{aligned}
$$

Onde, "IQR" representa o intervalo interquartílico (Q1- Q3) e os pontos fora destes limites são considerados valores discrepantes (outliers).

A modelagem via Equações de estimativa generalizadas (MEEG), proposta por Liang e Zeger., 1986, possui o intuito de estimar parâmetros de regressão quando os dados estão correlacionados. Em muitas ocasiões, apesar das variáveis estudadas serem independentes, a informação sobre uma determinada variável é coletada várias vezes ao longo do tempo, tornando-se observações correlacionadas (GOMES, 2015).

Liang e Zeger., 1986, desenvolveram o MEEG utilizando os modelos de quasi-verosimilhança (WENDDERBURN, 1974) para analisar dados longitudinais e necessita apenas da especificação da relação entre a média e a variância das observações (AGRANONIK, 2009). Contudo, define-se um modelo linear generalizado para cada instante de tempo, acrescentando uma função que faz ligação entre a parte aleatória e a parte sistemática:

$$
\text { nit }=g(\mu i t)
$$

O preditor linear é o vetor de parâmetros desconhecidos e de interesse e é a função de ligação. Utiliza-se a estrutura para a matriz de correlação do tipo simétrica ou permutável. As técnicas utilizadas foram aplicadas com o auxilio do software estatístico $\mathrm{R}$ 3.5.3. Utilizaram-se os pacotes geepack (Generalized Estimating Equation Package) e a função geeglm (Fit Generalized Estimating Equations) para a modelagem via equações de estimações generalizadas.

A definição do risco relativo (RR) é dada pela razão entre as probabilidades de sucesso de dois níveis da variável 
explicativa, (AGRESTI, 1996), sendo a equação do Risco Relativo dada por:

$$
R R=\frac{\pi 1}{\pi 2}
$$

Utilizou-se a estrutura para a matriz de correlação do tipo simétrica ou permutável, que assume:

$$
R(\alpha)=\left\{\begin{array}{l}
1 \text { se } t=t^{\prime} \\
\alpha \text { se } t \neq t^{\prime}
\end{array}\right.
$$

A equação 5 , deve ser utilizada quando se tem um modelo de efeitos aleatórios com intercepto aleatório para cada uma das variáveis, como exemplificado em Laird e Ware (1982)

Para fazer o preenchimento de falhas (2 meses no ano de 2015) utilizou-se o método de imputação múltipla via MICE (Multivariate Imputation by Chained Equation), cujo o mesmo é baseado no método de Monte Carlo via cadeia de Markov (LI et al., 2017). O MICE está disponível com pacote intitulado 'mice'.

\section{RESULTADOS E DISCUSSÃO}

A série temporal do número de casos por internações do Infarto Agudo do Miocárdio representada pela Figura 2 enfatiza três períodos de ascensão no número de casos para o primeiro período de 2012 a 2013, o segundo de 2014 a 2015 e o terceiro de 2016 a 2018, onde se observa um crescimento significativo, com magnitude ainda maior para os anos de 2016 e 2018. E um período de flutuação do número de casos em torno de um valor médio que vai de 2008 a 2010. Até o ano de 2012 o número de internações anuais eram inferiores a 350 casos. E a partir do ano de 2014 observou-se uma provável tendência de crescimento, a qual apresenta valores ultrapassando 600 casos.

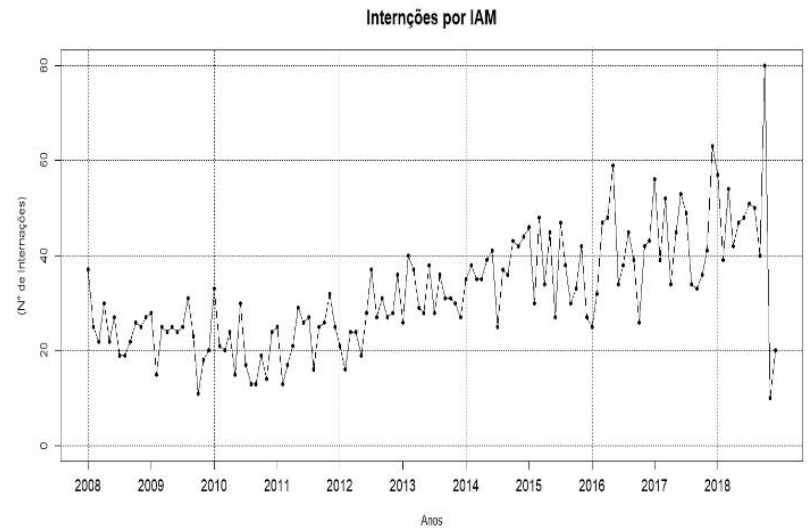

Figura 2 - Série temporal do número de casos por internação de Infarto do agudo miocárdio nos anos de 2008 a 2018, para a região de estudo. Fonte: autores (2021).
Estudos já comprovam que o acontecimento de doenças cardiovasculares está catalogado com a obesidade, alteração da pressão arterial, assim como hábitos de vida como o tabagismo, ingestão de álcool e o aumento do desconforto térmico (MANDÚ et al.,2019). A literatura recente indica compreender o aumento de condições térmicas como um dos fatores de risco, e ainda estimulam a realização de trabalho em regiões tropicais e subtropicais (PHUNG et al., 2016).

Diante da Figura 3, pode-se analisar a variabilidade das internações por IAM durante o período analisado, destaca-se o mês de março, o qual apresentou a maior variação dos números de internações tendo valores acima de 45 casos. Observa-se também que durante todo o ano, apenas no mês de outubro há presença de um outilier inferior, valor discrepante na série, onde no mesmo mês constatou-se que o menor número de casos estava abaixo de 35 internamentos. O mês de outubro também conta com presença de outline superior, que indica portanto, que em um dos anos do período estudado teve um número relevante de internações, assim comoo mês de dezembro.

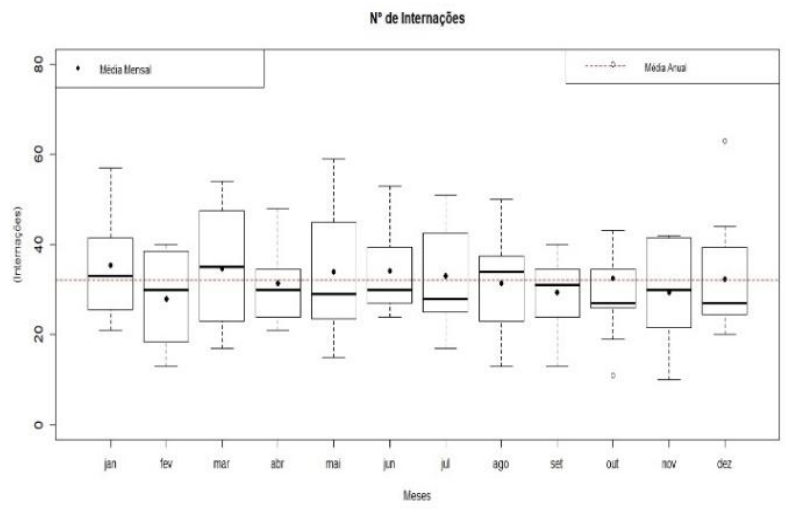

Figura 3 - Boxplot do número de internações mensais por IAM em Belém-PA no período de 2008 a 2018. Fonte: autores (2021).

Ao analisar o comportamento da série temporal das temperaturas mínima e máxima do ar em Belém do Pará no período estudado (Figura 4 e 5), constatou-se que a temperatura mínima apresentaram menores valores nos anos de 2013 e 2015, onde os valores se encontram abaixo de $23,0^{\circ} \mathrm{C}$ e maiores nos anos de 2011 e 2016 com valores acima de $24,0^{\circ} \mathrm{C}$.

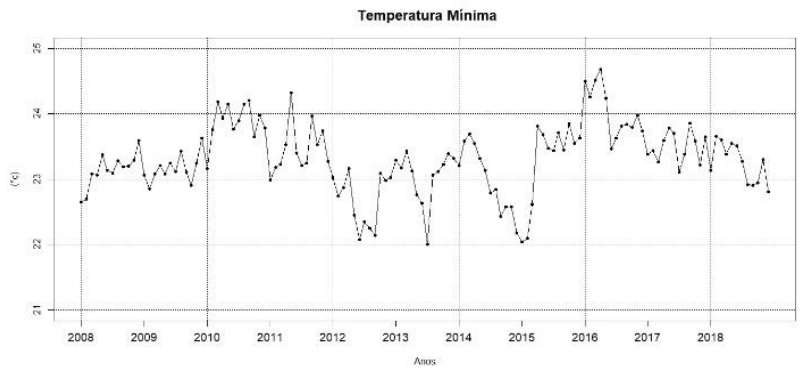

Figura 4 - Série temporal da temperatura mínima do ar nos anos de 2008 a 2018, para região de estudo. Fonte: autores. 
A série temporal da temperatura máxima (figura 5) em Belém apresenta valores superiores a $29,0{ }^{\circ} \mathrm{C}$ durante todo período analisado. Os valores abaixo de $30,0^{\circ} \mathrm{C}$ foram registrados no ano de 2008. Destacam-se os anos de 2015 e 2016, períodos que apresentam registros acima de $34,0{ }^{\circ} \mathrm{C}$.

No caso dessas temperaturas serem mais elevadas caracterizase por Belém estar situada graficamente próxima ao equador, por sua baixa altitude local, e a pequena troca térmica sendo relacionada com o regime de chuvas na região, em virtude das temperaturas máximas serem pouco enfatizadas com maior frequência e ocorrerem por ocasião do período mais chuvoso, enquanto que as mais elevadas atendem com o período menos chuvoso (BASTOS, 2002).

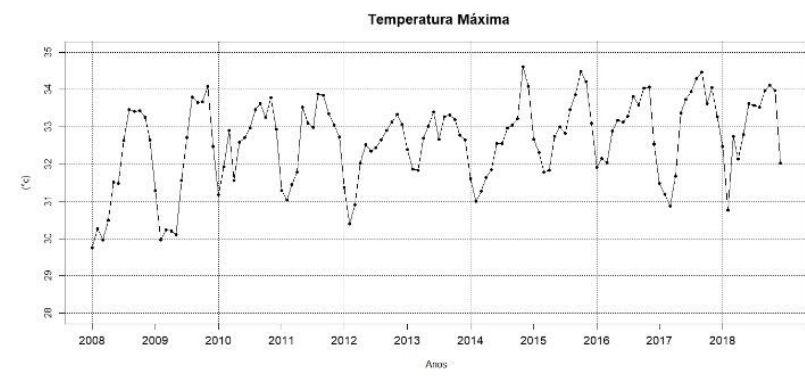

Figura 5 - Série temporal da temperatura máxima do ar nos anos de 2008 a 2018, para região de estudo. Fonte: autores (2021).

Na Figura 6 apresenta o gráfico que mosra a variabilidade mensal da temperatura mínima do ar, e é possível notar que essa variabilidade sazonal, possui como ciclo dominante, o semianual, com dois valores mínimos médios, janeiro $\left(23,13{ }^{\circ} \mathrm{C}\right)$ e julho $\left(23,08{ }^{\circ} \mathrm{C}\right)$, e dois valores médios máximos nos meses de maio $\left(23,41^{\circ} \mathrm{C}\right)$ e abril $\left(23,8^{\circ} \mathrm{C}\right)$. É possível observar a presença de diversos valores discrepantes, tanto inferiores quanto superiores.

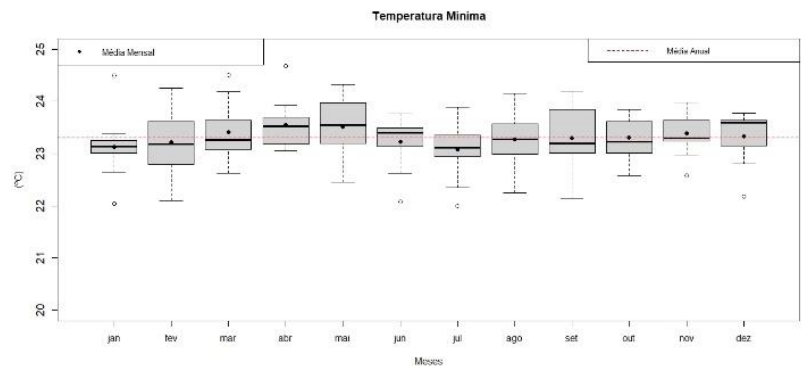

Figura 6 - Boxplot mensal da temperatura mínima do ar de 2008 a 2018, em Belém do Pará. Fonte: autores (2021).

Observa-se que, em Belém as temperaturas estão sempre altas, e isto está associado ao atent potencial de radiação solar incidente, a pesar de grande parte dessa energia ser convertida em calor atent de evaporação e outra parte convertida em calor sensível que é atribuido ao aquecimento do ar, (NECHET ,1984).

É possível observar que a temperatura máxima mensal (figura7) demonstra o ciclo anual bem definido, com menor valor médio em fevereiro $\left(31,17^{\circ} \mathrm{C}\right)$ e maior em novembro $\left(33,74^{\circ} \mathrm{C}\right)$. Apresenta também valores discrepantes nos meses de abril,julho e dezembro e outlines inferiores nos meses de janeiro, março e maio.

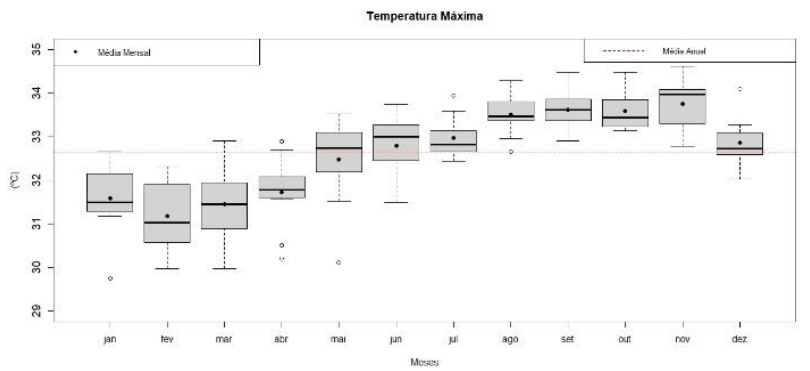

Figura 7 - Boxplot mensal da temperatura máxima do ar de 2008 a 2018, em Belém do Pará. Fonte: autores.

A figura 8 , mostra a serie anual da precipitação e é notório que os anos de 2008, 2011 e 2012 estiveram sob a influência do fenômeno La Niña que aumentam as chuvas na cidade de Belém (OLIVEIRA et al., 2016). Os anos de 2010 e 2015 mostram menores valores, que tiveram influência pelo fenômeno El Niño que provoca a redução dos índices pluviométricos na região amazônica, a "famosa chuva da tarde" em Belém, tem grande contribuição para os totais mensais, sendo as $16 \mathrm{~h} 00$ o horário de maior contribuição, no período entre a madrugada e início da manhã, os valores de contribuição são mínimos (MORAES, 2017).

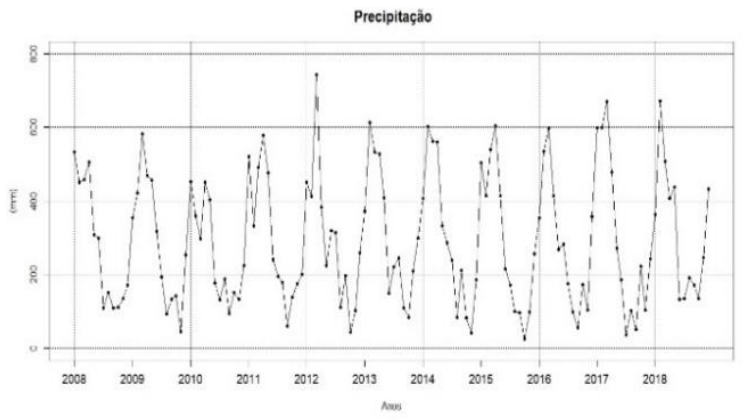

Figura 8 - Série temporal da precipitação acumulada $(\mathrm{mm})$ nos anos de 2008 a 2018, para a região de estudo. Fonte: autores (2021).

Entre os anos de 2008 a 2018 nota-se maior variabilidade da precipitação pluvial nos meses de janeiro, março, junho e agosto (Figura 9). O período de maior pluviosidade ocorreu nos meses de janeiro a maio, com ênfase no mês de março tendo seu valor máximo de $543,5 \mathrm{~mm}$ e com maior estiagem no período de julho a novembro, com menor valor no mês de setembro, totalizando $118 \mathrm{~mm}$.

Segundo Vianello; Alves (2013) dentro das circunstâncias locais, pode-se descrever que a precipitação em Belém/PA como resultante das seguintes situações: de dezembro a maio, época mais chuvosa, a precipitação é originada pela Zona de Convergência Intertropical (ZCIT), e pelos efeitos de mesoescala, como as linhas de instabilidades que se formam na costa Atlântica da Guianas e do Pará, e propagam-se para o oeste como uma linha de cumulonimbus (BASTOS et al., 2002, p. 14). 


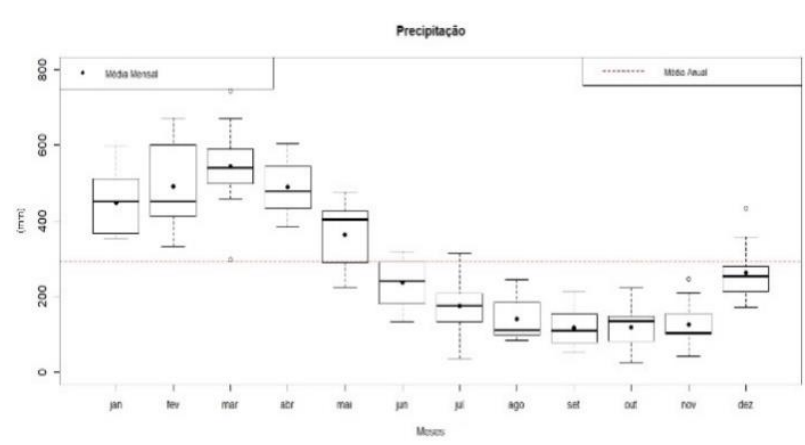

Figura 9 - Boxplot da precipitação pluvial $(\mathrm{mm})$ por mês 2008 a 2018, em Belém do Pará. Fonte: autores (2021).

O conceito de estações do ano para a regiao da Amazônia é diferente das demais regiões do Brasil. Em geral, tem-se o "período seco" (de julho a outubro) e o "período chuvoso" (de dezembro a maio), sendo os meses de junho e novembro, os períodos de transição.

No comportamento da umidade relativa do ar (Figura 10 ) é possível observar no período estudado, sendo assim caracterizando a umidade relativa constantemente elevada na cidade de Belém do Pará. Dentre o período analisado, destacamse os anos de 2008, 2009, 2017 e 2018 como os maiores registros.

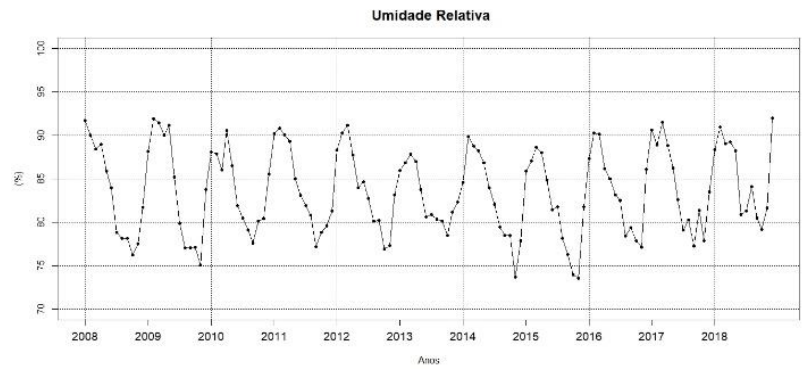

Figura 10 - Série temporal da umidade relativa do ar (\%) nos anos de 2008 a 2018, para a região de estudo. Fonte: autores (2021).

Na Figura 11, encontra-se o gráfico de variabilidade mensal da variável umidade relativa do ar, e é possível observar que o maior valor está presente no mês de fevereiro $(89,51 \%)$ e menor valor em novembro $(77,72 \%)$, com período de transição de abril a maio para períodos de esitiagem, como transição para época mais chuvosa.

Esse elemento apresenta estreita relação com a distribuição das chuvas e com a temperatura do ar no decorrer dos anos. A distribuição mensal da umidade relativa relaciona-se com a distribuição das chuvas, e que os valores mais elevados de umidade ocorreram nos meses de maior pluviosidade.

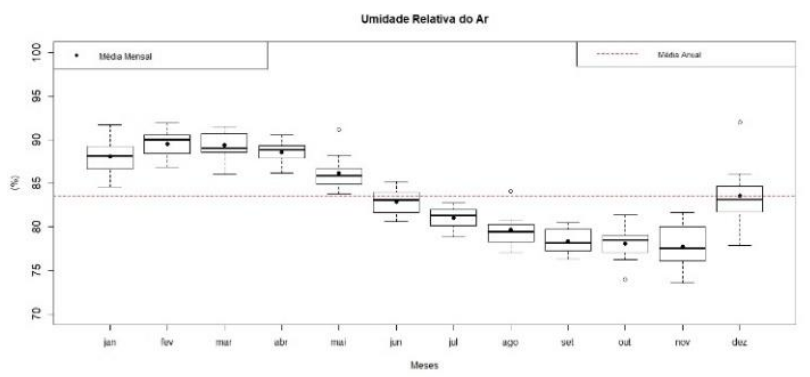

Figura 11 - Boxplot da umidade relativa do ar (\%) por mês 2008 a 2018, em Belém do Pará. Fonte: autores (2021).

Verifica-se que a variabilidade da velocidade do vento que está representada na figura 12 , apresenta maiores valores nos anos de 2008 a 2010, destacando o ano de 2009 onde foi registrado o maior valor de $2,5(\mathrm{~m} / \mathrm{s})$ e menores valores nos anos de 2012, 2016 e 2018 sendo inferiores a $1,5(\mathrm{~m} / \mathrm{s})$.

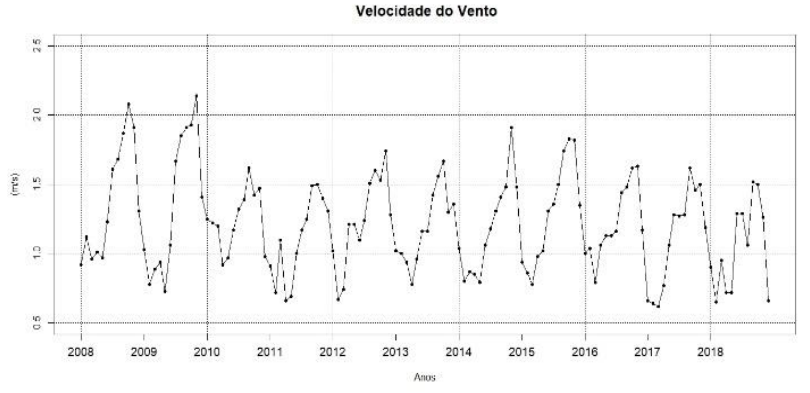

Figura 12 - Série temporal da velocidade do vento nos anos de 2008 a 2018, PA. Fonte: autores (2021).

Observa-se para Belém/PA que existe a predominância de calmaria $(83,3 \%)$, seguido de ventos fracos com classes de velocidade de 1,0 a $2,0 \mathrm{~m} / \mathrm{s}$ (3,9\%), categorizados como circulação de brisa leve, de acordo com a escala Beaufort (WMO, 2000). De acordo com a World Meteorological Organization (WMO) a situação de calmaria é considerada quando a magnitude do vento se apresenta menor a 1 nó $0,515(\mathrm{~m} / \mathrm{s})$. Sazonalmente, ocorre uma variação das velocidades médias, entre 0,90 e 1,50 $(\mathrm{m} / \mathrm{s})$.

A variável velocidade do vento (Figura 13) mostra também um ciclo anual bem definido, onde é possível observar que no mês de novembro apresenta o maior valor registrado 1,64 (m/s), e valores inferiores no mês de fevereiro chegando $0,86(\mathrm{~m} / \mathrm{s})$. 


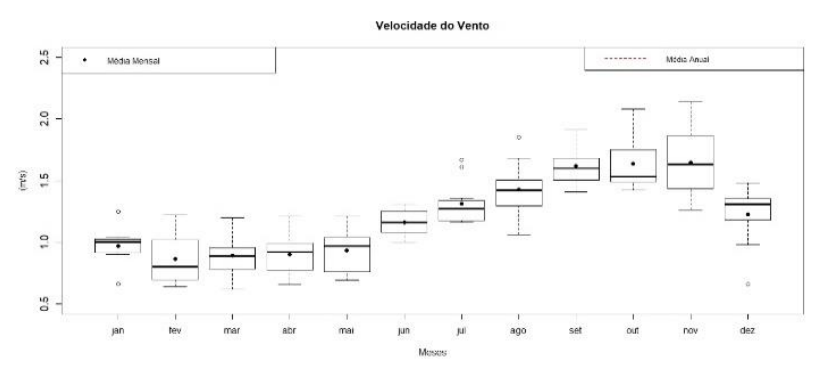

Figura 13 - Boxplot da velocidade do vento por mês 2008 a 2018 , em Belém do Pará. Fonte: autores (2021).

Tabela 1: Modelo Marginal de Equações de Estimação Generalizada (GEE), entre variáveis meteorológicas e Internações por Infarto Agudo do Miocárdio, em Belém do Pará, 2008 - 2018. Fonte: autores (2021).

\begin{tabular}{llccc}
\hline Variáveis & Estimativa & Erro & p-valor & RR* $^{*}$ \\
\hline Temperatura Máxima & $\mathbf{0 , 2 4}$ & $\mathbf{0 , 0 6 0}$ & $<\mathbf{0 , 0 0 1}$ & $\mathbf{1 , 2 7}$ \\
Velocidade do Vento & $\mathbf{- 0 , 3 5}$ & $\mathbf{0 , 1 8 7}$ & $<\mathbf{0 , 1}$ & $\mathbf{0 , 7 0}$
\end{tabular}

Legenda: Significância 5\%, RR*= Risco relativo.

O resultado da modelagem via equações de estimação generalizadas é observado na Tabela 1, onde se constata associação estatística significativa entre as internações por Infarto Agúdo do Miocárdio e as variáveis meteorológicas: Temperatura máxima do ar e velocidade do vento. Com isso, aceita-se a hipótese que a sinergia entre valores altos da temperatura do ar, contribui para o aumento do número de internações por IAM, já a relação entre velocidade do vento e a doença ocorre de forma inversa. Destaca-se que por se tratar da temperatura máxima esses efeitos negativos podem ser maiores no período da tarde já que é o período onde se tem as maiores temperatura na cidade em estudo.

Entre as outras variáveis meteorlogicas analisadas: umidade relativa, precipitação e temperatura mínima do ar, não apresentaram relações significativas em relação as internações. Porém, uma vez que os seres humanos por estarem permanentemente em contato com a atmosfera, alteram a sua dinâmica e desta forma tornam-se vítimas da influência que exercem sobre o ambiente. Com isso, algumas doenças, surgem ou desenvolvem-se devido às variações naturais ou antrópicas, frequentes dos elementos climáticos.

Os resultados deste trabalho corroboram com os de keatinge (1986) e Sharovsky (2001), onde os autores afirmam a existencia de relação direta entre a temperature máxima do ar e o número de casos de IAM, isto é quando a um aumento brusco na temperatura do ar o número de internações crescem.

De acordo com Keatinge e col.11., 1986, o aumento da temperatura acarreta um suor rigoroso que leva a uma diminuição do volume plasmático e a queda da pressão arterial, além de aumentar a viscosidade do sangue, do número de hamácias e de plaquetas, podendo envolver-se em ateromas que interropem o fluxo sanguineo para os tecidos, causando então, IAM, conforme o orgão afetado.
Outra contribuição relevante nesta área foi o estudo de Murara et al., 2010, onde denomina-se que as variações de tipos de tempo influenciam a temperatura corporal, dado que, ativam os mecanismos naturais (homeostáticos) humanos para o domínio da estabilidade entre o meio externo e o organismo interno. Uma vez que o corpo humano saudável apresenta maior eficácia a uma temperatura de $37^{\circ} \mathrm{C}$ (Equilíbrio Térmico). O organismo humano responde a esse processo ativando o funcionamento da vasoconstricção e vasodilatação, sobrecarga ou menor fluxo dos vasos sanguíneos e do coração (TORTORA, 2000). Sendo assim, isso ocorre geralmente com o aumento da tensão arterial, sendo capaz de proceder na maior incidência de doenças circulatórias.

\section{CONSIDERAÇÕES FINAIS}

Foram registrados cerca de 4242 casos de internações por Infarto agudo do miocárdio no período em estudo e foi observado que nos anos de 2016 e 2018 houve um aumento considerável na taxa de internações. A modelagem apresentou associações significativas entre as variáveis independentes (temperatura máxima e velocidade do vento) e dependente (internações). Portanto, em Belém do Pará entre os períodos de 2008 a 2018 deu-se que a alteração da temperatura máxima e a velocidade do vento contribuíram para um maior risco de internações da população por Infarto agudo do miocárdio.

\section{REFERÊNCIAS}

ARAÚJO, Adilson Ribeiro de et al. O tempo e o clima: análise da dinâmica climática e das notícias veiculadas pelos meios de comunicação em Cuiabá, Mato Grosso. 2018.

AGRESTI, A. An introduction to categorical data analysis. New York: Wiley, 1996. 
AGRANONIK, Marilyn. Equações de estimação generalizadas (GEE): aplicação em estudo sobre mortalidade neonatal em gemelares de Porto Alegre, RS (1995-2007). 2009.

ATAQUE CARDÍACO (INFARTO). Ministério da saúde, 2018. Disponível em: <https://bvsms.saude.gov.br/dicas-emsaude/2779-ataque-cardiaco infarto\#: :text=0\%20infarto $\% 20 \mathrm{do} \% 20 \mathrm{mioc} \% \mathrm{C} 3 \% \mathrm{~A} 1 \mathrm{rdio}$ $\% 2 \mathrm{C} \% 20 \mathrm{ou}, \mathrm{de} \% 20$ forma\%20s\%C3\%BAbita\%20e\%20inte nsa.>.Acesso em: 06/05/2021.

BACELAR, A.C. Infarto do Miocárdio. Hospital Israelita Albert Einstein. 26 de abril de 2012. Disponível em: https://www.einstein.br/especialidades/cardiologia/doencas - sintomas/infarto-do-miocardio. Acesso em 16 de novembro 2019.

BASTOS, Therezinha Xavier et al. Aspectos climáticos de Belém nos últimos cem anos. Embrapa Amazônia OrientalDocumentos (INFOTECA-E), 2002.

BRASIL. Ministério da saúde. Dia mundial do coração. Brasília: Ministério da saúde, 2019..

DATASUS. Ministério da Saúde, Brasil, 2018. Disponível em. Atualizações - DATASUS (saude.gov.br) Acesso em $15 \mathrm{de}$ novembro de 2019.

DILAVERIS, P. et al. CLimate Impacts on Myocardial infarction deaths in the Athens Territory: the CLIMATE study. Heart, v. 92, n. 12, p. 1747-1751, 2006.

FIGUEROA, Silvio N.; NOBRE, Carlos A. Precipitation over central and western tropical South America. Climanalise, v. 5, n. 6, p. 36-45, 1990.

GOERRE, Stefan et al. Impact of weather and climate on the incidence of acute coronary syndromes. International journal of cardiology, v.118, n. 1, p. 36-40, 2007.

GOMES, A. C. S. Três alternativas estocásticas para modelar morbimortalidade por doenças respiratórias $e$ cardiovasculares via variáveis atmosféricas. 2015.

HEYER, Howard E.; TENG, H. C.; BARRIS, William. The increased frequency of acute myocardial infarction during summer months in a warm climate: A study of 1,386 cases from Dallas, Texas. American heart journal, v. 45, n. 5, p. 741-748, 1953.

IBGE. Instituto Brasileiro de Geografia e Estatística. Acesso em 17 de novembro de 2019.

INMET.http://www.inmet.gov.br/portal/index.php? $r=b d m e p / b d$ mep. Acesso em 17 de novembro de 2019 às 16:52.

IKEFUTI, Priscilla Venâncio. Associação entre variáveis meteorológicas, índice climático, fatores socioeconômicos e mortalidade por doenças do aparelho circulatório (acidente vascular cerebral e embolia pulmonar) no município de São Paulo-SP. Tese de Doutorado. Universidade de São Paulo, 2017.
Instruments and Methods of Observation, Geneva, Instruments and Observing Methods Report n. 74, WMO/TD-n. 1028, 72, 2000 .

KEATINGE, W. R., Coleshaw, S. R., Easton, J. C., Cotter, F., Mattock, M. B., \& Chelliah, R. (1986). Increased platelet and red cell counts, blood viscosity, and plasma cholesterol levels during heat stress, and mortality from coronary and cerebral thrombosis. The American journal of medicine, 81(5), 795-800.

LAIRD, N. M.; WARE, J. H. Random-effects models for longitudinal data. Biometrics, v. 38, n. 4, p. 963-974, 1982.

LEE, Jang Hoon et al. Influência do clima nas internações diárias por Infarto Agudo do Miocárdio (do Registro de Infarto Agudo do Miocárdio da Coréia). Revista Internacional de Cardiologia, v. 144, n. 1, p. 16-21, 2010.

LIANG, K. -Y.; ZEGER, S. L. Longitudinal data analysis using generalized linear models. Biometrika, v. 73, n. 1, p. 13-22, 1986.

LIMA, Areta Cristina et al. DATASUS: O uso dos Sistemas de Informação na Saúde Pública. Refas-Revista Fatec Zona Sul, v. 1, n. 3, p. 16-31, 2015.

LI, P.; STUART, E.A.; ALLISON, D.B. Multiple imputation a flexible tool for handing missing data, Jama, v.314, n.8, p.1966-1967, 2017. https://doi.org/10.1001/jama.2015.15281

MANDÚ, Tiago Bentes et al. Associação entre o indice de calor e internações por infarto agúdo do miocárdio em Manausam. 2019.

MARENGO, J. A.; OLIVEIRA, GS de. Impactos do fenômeno La Niña no tempo e clima do Brasil: desenvolvimento e intensificação do La Niña 1998/1999. In: Congresso Brasileiro De Meteorologia. 1998.

MEDEIROS, T. L. F. D., Andrade, P. C. N. S. D., Davim, R. M. B., \& Santos, N. M. G. D. (2018). Mortalidade por infarto agudo do miocárdio. Rev. enferm. UFPE on line, 565-573.

MORABITO, Marco et al. Relationship between climate and myocardial infarction: a biometeorological approach. International Journal of Cardiology, v. 105, n. 3, p. 288-293, 2005.

MORAES, D. S. dos S. Contribuição das chuvas do período da tarde em Belém do Pará para os totais mensais e possíveis relações com a média climatológica. 2017. 100 f. Dissertação (Mestrado em Meteorologia) - Programa de Pós-Graduação em Meteorologia, Centro de Tecnologia e Recursos Naturais, Universidade Federal de Campina Grande, Paraíba, Brasil, 2017.

MINISTÉRIO DA SAÚDE. http://www.saude.gov.br/vigilanciaem-saude/vigilancia-ambiental/vigidesastres/mudancasclimaticas. Acesso em 22 de novembro de 2019. 
MURARA, P. G., Coelho, M. D. S. Z. S., \& Amorim, M. C. D. C. T. (2010). Análise da influência meteorológica nas internações por doenças cardiovasculares. Caderno Prudentino de Geografia, 1(32), 53-66.

NECHET, D. Ocorrência de um tornado em Belém-PA. Belém:Seção de Meteorologia do Serviço Regional de Proteção ao Vôo de Belém, 1984a. 9p. Mimeografado.

Oliveira, G. B; Alcântara, C. R.; Souza, E. P. 2016. Caracterização de perfil de vento no ambiente de formação das linhas de instabilidade amazônicas. Ciência e Natura, Santa Maria v.38 n.1, Jan.- Abr. p. 393 - 403, DOI:10.5902/2179-460X18337.

OLIVEIRA, Joelma Cristine Figueredo de et al. Estudo da influência das ilhas de amenidade nos centros urbanos: o caso do Recife. 2019.

OLIVEIRA, M. S. P. D.; COUTURIER, Guy; BESERRA, Paulo. Biologia da polinização da palmeira tucumã (Astrocaryum vulgare Mart.) em Belém, Pará, Brasil. Embrapa Amazônia Oriental-Artigo em periódico indexado (ALICE), 2003.

PEREIRA, H.S., FREITAS E SILVA, P. M., DANTAS, R. T., ARAÚJO, R. A. F. Correlação de variáveis meteorológicas com infarto agudo do miocárdio. 2007. Disponível em: http://www.sbmet.org.br/sic2011/arq/90856437217219085 643721.pdf. Acesso em: NOVEMBRO/2019.

PEREIRA, Heronides dos Santos et al. Influência e correlação de variáveis meteorológicas com infarto agudo do miocárdio e diabetes mellitus. 2012.

PHUNG, Dung et al. Ambient temperature and risk of cardiovascular hospitalization: An updated systematic review and meta-analysis. Science of The Total Environment, v. 550, p. 1084-1102, 2016.
Rumel, D., Riedel, L. F., Latorre, M. D. R., \& Duncan, B. B. (1993). Infarto do miocárdio e acidente vascular cerebral associados à alta temperatura e monóxido de carbono em área metropolitana do sudeste do Brasil. Revista de saúde pública, 27, 15-22.

SANTOS, J dos, et al. Mortalidade por Infarto Agudo do Miocárdio no Brasil e suas regiões geográficas: análise do efeito da idade-período-coorte. Ciência \& Saúde Coletiva, v. 23, p. 1621-1634, 2018.

SHAROVSKY, R. (2001). Efeitos da temperatura e poluição do ar na mortalidade por infarto agudo do miocárdio no município de São Paulo (Doctoral dissertation).

TENG, H. C.; HEYER, Howard E. The relationship between sudden changes in weather and the occurrence of acute myocardial infarction. American heart journal, v. 49, n. 1, p. 9-20, 1955

TROPICAL, Pacífico. variações do ciclo anual da temperatura da superfície do mar no. Revista Brasileira de Meteorologia, $v$. 25, n. 2, p. 237-247, 2010.

TORTORA, G. J. O Sistema Circulatório. In: Corpo Humano: fundamentos de anatomia e fisiologia. Porto Alegre: Armed Editora, 2000.

VIANELlO, R. L.; ALVES, A. R. Meteorologia básica $e$ aplicações. 2. ed. Viçosa: Editora UFV, 2013.

WEDDERBURN, RWM. Quasi-likelihood functions, generalized linear models, and the Gauss-Newton method. Biometrika, v. 61, n. 3, p. 439-447, 1974.

WMO (World Meteorological Organization). Wind measurements: Potential wind speed derived from wind speed fluctuations measurements, and the representativity of wind stations (J.P. van der Meulen). In: Proceedings of the WMO Technical Conference on Meteorological and Environmental.

Recebido em: 25/03/2021

Aceito para publicação em: 20/07/2021 